\title{
PSFC/JA-04-41
}

\section{Overview of the Alcator C-Mod Program}

Greenwald, M., Andelin, D., Basse, N., Bernabei, S. ${ }^{2}$, Bonoli, P., Böse, B., Boswell, C. ${ }^{5}$, Bravenec, R. ${ }^{1}$, Carreras, B. ${ }^{3}$, Cziegler, I. ${ }^{8}$, Edlund, E., Ernst, D., Fasoli, C. ${ }^{7}$, Ferrara, M., Fiore, C.,Granetz, R., Grulke, O. ${ }^{4}$, Hender, T. ${ }^{5}$,

Hosea, J. ${ }^{2}$, Howell, D.H. ${ }^{11}$, Hubbard, A., Hughes, J., Hutchinson, I., Ince-Cushman, A., Irby, J., LaBombard, B., LaHaye, R. ${ }^{11}$, Lin, L., Lin, Y., Lipschultz, B., Liptac, J., Lisgo, S. ${ }^{6}$, Lynn, A. ${ }^{1}$, Marmar, E., Marr, K., Mikkelsen, D.R. ${ }^{2}$, McDermott, R., Mossessian, D., Parisot, A., Parker, R., Phillips, C. ${ }^{2}$, Phillips, P. ${ }^{1}$, Porkolab, M., Redi, M. ${ }^{2}$, Rice, J., Rowan, W. ${ }^{1}$, Sampsell, M. ${ }^{1}$, Schilling, G. ${ }^{2}$, Scott, S. ${ }^{2}$, Scoville, J.T. ${ }^{11}$, Smick, N., Snipes, J., Stangeby, P. ${ }^{6}$, Tang, V., Terry, J., Ulrickson, M., Wallace, G., Wilson, J. ${ }^{2}$, Wright, J., Wolfe, S., Wukitch, S., Youngblood, B., Yuh, H., Zhurovich, K., Zweben, S. ${ }^{2}$

November 16, 2004

Massachusetts Institute of Technology, Cambridge, MA USA

${ }^{1}$ University of Texas, Austin, TX, USA

${ }^{2}$ Princeton Plasma Physics Laboratory, Princeton, NJ, USA

${ }^{3}$ Oak Ridge National Laboratory, Oak Ridge, TN, USA

${ }^{4}$ Max Planck Institute for Plasma Physics, Greifswald, Germany

${ }^{5}$ JET Joint Undertaking, Culham, UK

${ }^{6}$ University of Toronto, Ontario, Canada

${ }^{7}$ CRPP $\backslash 226$ EPFL, Lausanne, Switzerland

${ }^{8}$ Eotovos Lorand University, Budapest, Hungary

${ }^{9}$ Sandia National Laboratory, NM, USA

${ }^{10}$ University of Wisconsin, Madison, USA

${ }^{11}$ General Atomics, San Diego, USA

This work was supported by the U.S. Department of Energy, Cooperative Grant No. DE-FC02-99ER54512. Reproduction, translation, publication, use and disposal, in whole or in part, by or for the United States government is permitted.

Submitted for publication to the Proceedings of the $20^{\text {th }}$ IAEA Fusion Energy Conference. 


\title{
Overview of the Alcator C-Mod Program
}

M. Greenwald 1), D. Andelin 1), N. Basse 1), S. Bernabei 3), P. Bonoli 1), B. Böse 1), C. Boswell 6), R. Bravenec 2), B. Carreras 4), I. Cziegler 9), E. Edlund 1), D. Ernst 1), C. Fasoli 8), M. Ferrara 1), C. Fiore 1), R. Granetz 1), O. Grulke 5), T. Hender 6), J. Hosea 3), D. H. Howell 12), A. Hubbard 1), J. Hughes 1), I. Hutchinson 1), A. Ince-Cushman 1), J. Irby 1), B. LaBombard 1), R. LaHaye 12), L. Lin 1), Y. Lin 1), B. Lipschultz 1), J. Liptac 1), S. Lisgo 7), A. Lynn 2), E. Marmar 1), K. Marr 1), D.R. Mikkelsen 3), R. McDermott 1), D. Mossessian 1), A. Parisot 1), R. Parker 1), C. Phillips 3), P. Phillips 2), M. Porkolab 1), M. Redi 3), J. Rice 1), W. Rowan 2), M. Sampsell 2), G. Schilling 3), S. Scott 3), J.T. Scoville 12), N. Smick 1), J. Snipes 1), P. Stangeby 7), V. Tang 1), J. Terry 1), M. Ulrickson 10), G. Wallace, J. Wilson 3), J. Wright 1), S. Wolfe 1), S. Wukitch 1), B. Youngblood 1), H. Yuh 1), K. Zhurovich 1), S. Zweben 3)

1) Massachusetts Institute of Technology, Cambridge, MA, USA

2) University of Texas, Austin, TX, USA

3) Princeton Plasma Physics Laboratory, Princeton, NJ, USA

4) Oak Ridge National Laboratory, Oak Ridge, TN, USA

5) Max Planck Institute for Plasma Physics, Greifswald, Germany

6) JET Joint Undertaking, Culham, UK

7) University of Toronto, Ontario, Canada

8) CRPP - EPFL, Lausanne, Switzerland

9) Eotovos Lorand University, Budapest, Hungary

10) Sandia National Laboratory, NM, USA

11) University of Wisconsin, Madison, USA

12) General Atomics, San Diego, USA

e-mail contact of main author: g@psfc.mit.edu

\begin{abstract}
Research on the Alcator C-Mod tokamak has emphasized RF heating, self-generated flows, momentum transport, scrape-off layer turbulence and transport and the physics of transport barrier transitions, stability and control. The machine operates with $\mathrm{P}_{\mathrm{RF}}$ up to $6 \mathrm{MW}$ corresponding to power densities on the antenna of 10 $\mathrm{MW} / \mathrm{m}^{2}$. Analysis of rotation profile evolution, produced in the absence of external drive, allows transport of angular momentum in the plasma core to be computed and compared between various operating regimes. Momentum is clearly seen diffusing and convecting from the plasma edge on time scales similar to the energy confinement time and much faster than neo-classical transport. Scrape-off layer (SOL) turbulence and transport have been studied with fast scanning electrostatic probes, situated at several poloidal locations and with gas puff imaging. Strong poloidal asymmetries are found in profiles and fluctuations, confirming the essential ballooning character of the turbulence and transport. Plasma topology has a dominant effect on the magnitude and direction of both core rotation and SOL flows. The correlation of self-generated plasma flows and topology has led to a novel explanation for the dependence of the H-mode power threshold on the $\nabla \mathrm{B}$ drift direction. Research into internal transport barriers (ITB) has focused on control of the barrier strength, and location. The foot of the barrier could be moved to larger minor radius by lowering $\mathrm{q}$ or $\mathrm{B}_{\mathrm{T}}$. The barriers, which are produced in C-Mod by off-axis $\mathrm{RF}$ heating, can be weakened by the application of on-axis power. Gyro-kinetic simulations suggest that the control mechanism is due to the temperature dependence of trapped electron modes (TEM) which are destabilized by the large density gradients. A set of non-axisymmetric coils was installed allowing intrinsic error fields to be measured and compensated. These also enabled the determination of the mode locking threshold and, by comparison with data from other machines, provided the first direct measurement of size scaling for the threshold. The installation of a new inboard limiter resulted in the reduction of halo currents following disruptions. This effect can be understood in terms of the change in plasma contact with the altered geometry during vertical displacement of the plasma column. Unstable Alfven eigenmodes (AE) were observed in low-density high-power ICRF heated plasmas. The damping rate of stable AEs was investigated with a pair of active MHD antennae.
\end{abstract}




\section{Introduction}

Alcator C-Mod is a compact high-field tokamak that can run high performance plasmas at densities and collisionality up to an order of magnitude higher than machines of standard design. The collision frequency relative to confinement is much more prototypical of a reactor than in other devices, that is, in most regimes $\tau_{\mathrm{ei}} \gg \tau_{\mathrm{E}}$ which results in close coupling of electrons and ions. In the scrape-off layer (SOL) and divertor, ions, neutrals and photons are all in collisional regimes typical of large-scale reactors like ITER. By running at relatively low density and high power, C-Mod can also be paired with larger lower-field devices to perform dimensionless identity and dimensionless scaling experiments. These have already yielded important insights into core, pedestal and SOL physics. C-Mod uses ICRF rather than neutral beams as its principal auxiliary heating method, thus the heating source is not associated with strong particle, momentum, or current sources. Scrape-off layer power densities are very high and typical of those in a reactor. Plasma facing components are made of high-Z metals which are of interest for reactors because of their superior properties relative to erosion, tritium co-deposition and power handling. Metallic walls have lower hydrogen retention properties than graphite, with the result that $\mathrm{C}$-Mod tends to run close to equilibrium with respect to wall pumping and recycling. A new inner divertor was installed recently which allowed operation at high-triangularity and which strengthened the inner wall against disruption loads. This development, combined with the installation of non-axisymmetric correction coils enabled operation with $\mathrm{I}_{\mathrm{P}}$ up to $2 \mathrm{MA}$.

\section{ICRF Heating and Current Drive}

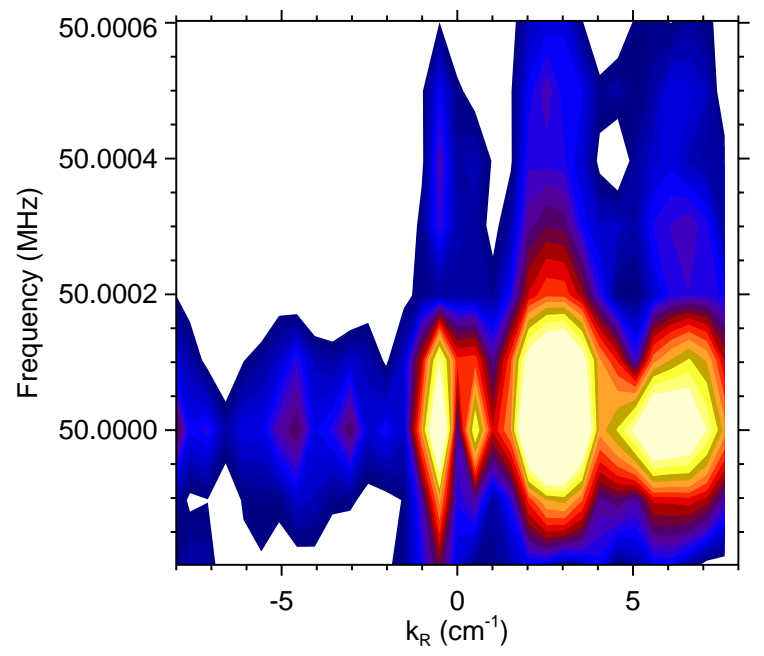

Fig 1. Density fluctuations, measured with the PCI diagnostic, show all three waves (fast-wave, IBW, ICW) predicted by simulations of the mode conversion process in a D-He3 mixture at $50 \mathrm{MHz}$. (The analysis process results in some mixing of the mode conversion spectra.)

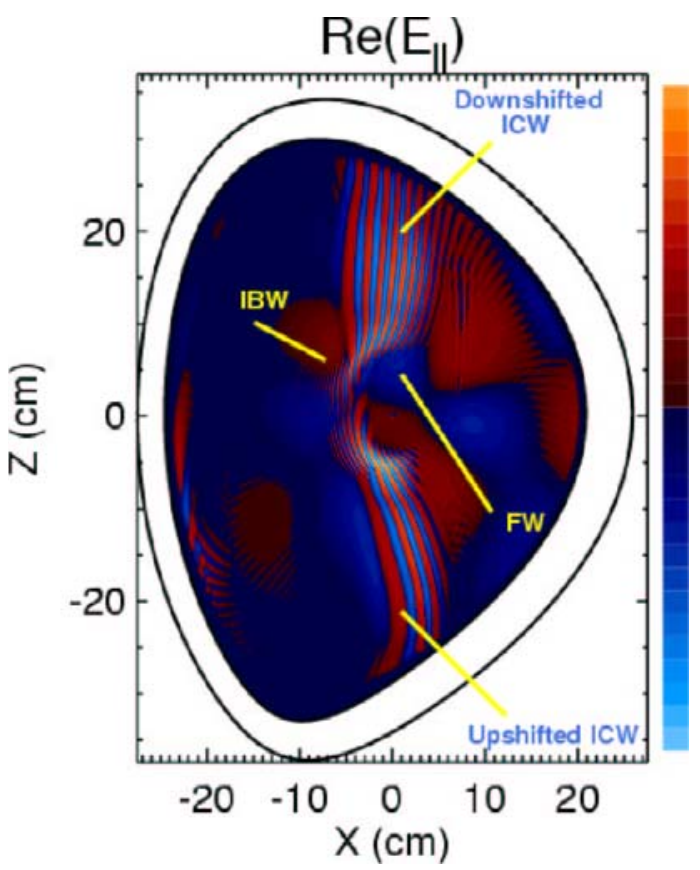

Fig 2. Simulations of the mode conversion process with the TORIC code show the forward propagating IBW as well as the backward propagating ICW. 
Auxiliary heating in Alcator C-Mod is via RF in the Ion Cyclotron Range of Frequencies (ICRF). Three antennae are available - two 2-strap antennae connected to transmitters at 80 $\mathrm{MHz}$ and a 4-strap antenna which is connected to a tunable source, useful from 50-80 MHz. The principal heating scenarios employed are hydrogen minority in deuterium at toroidal fields from $3.6-6.4 \mathrm{~T}$ and helium 3 minority at $7-8 \mathrm{~T}$. Net power coupled to the plasma has reached $6 \mathrm{MW}$ for up to 0.4 seconds with launched power density on the 4-strap antenna up to 10 $\mathrm{MW} / \mathrm{m}^{2}$ [1]. Good phase control has been demonstrated and efficient heating with low impurity generation has been shown for both heating and current drive phasing. Indirect evidence suggests that plasma current on the order of $100 \mathrm{kA}$ was driven. Mode conversion scenarios have also been investigated and the first measurements and simulations of the mode converted ion cyclotron wave (ICW) made [2,3]. This wave, first predicted by Perkins in 1977 [4], shows up as a weakly damped mode on the low field side of the ion-ion hybrid layer and propagates in the positive $\mathrm{R}$ direction (opposite to the launched wave.) Its wavelength is intermediate between the fast wave (FW) and the mode converted ion Bernstein wave (IBW) which both propagate in the negative $\mathrm{R}$ direction. Figure 1 shows measurements of all three of these waves using phasecontrast imaging (PCI). With a parallel version of the TORIC ICRF code, simulations with sufficient spatial resolution to resolve the mode conversion process have been carried out (figure 2) [5]. These simulations show that the partition of power between the IBW and ICW depend on the magnitude of the poloidal field; at high current the power into the ICW can exceed the IBW. Quantitative comparisons of the mode conversion heating profiles have been made and show good agreement only if sufficiently high mode numbers are included in the simulation.
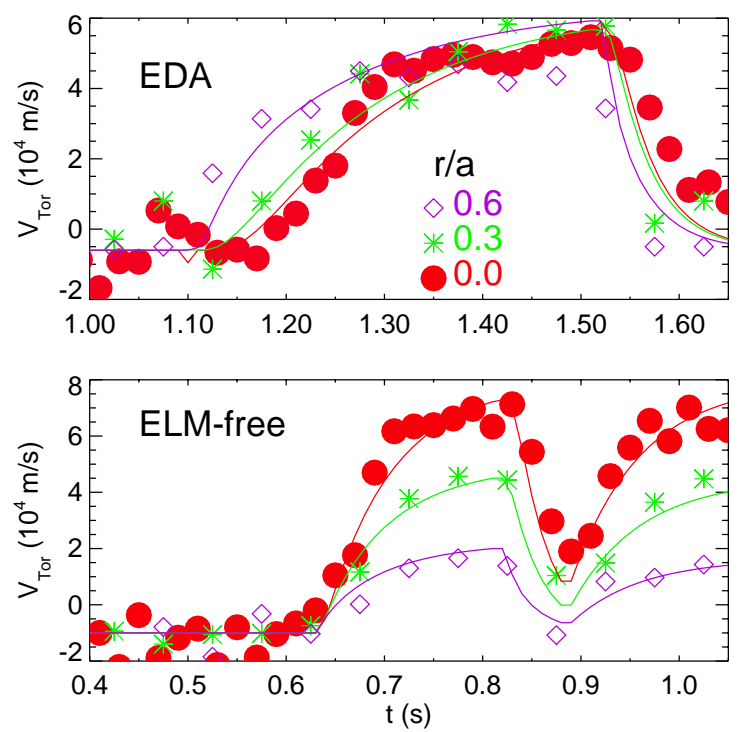

Fig 3. Traces of core toroidal rotation through an $\mathrm{L}-\mathrm{H}-\mathrm{L}$ cycle are plotted for different radii (symbols). For EDA $\mathrm{H}$ modes, the data can be modelled (lines) as a diffusive process where momentum is transported from the edge. ELM-free $\mathrm{H}$ modes show a large inward convection of momentum.

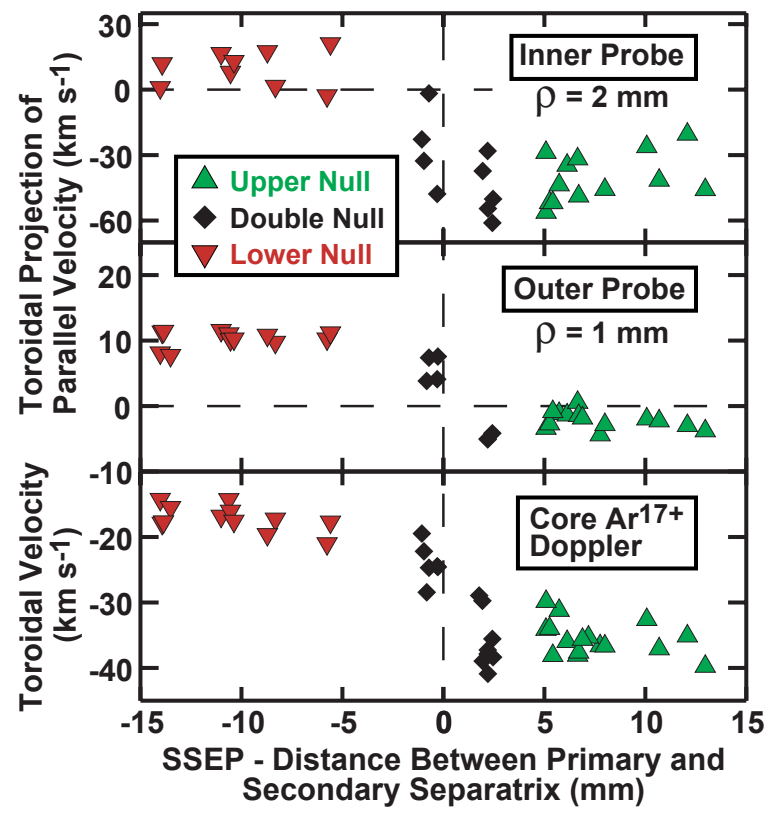

Fig 4. Toroidal velocity components are plotted for a set of discharges spanning from lower single null (ssep <0) through balanced double null (ssep $\sim 0)$ to upper single null (ssep > 0). Both core and SOL values show similar, extreme sensitivity to this parameter, suggesting a common origin. 
Implications for flow drive of this newly reported mode-conversion process are also being evaluated [6], with early experiments showing some evidence of enhanced plasma rotation.

\section{Spontaneous Rotation and Momentum Transport}

Observations of strong rotation, produced in the absence of an external torque in C-Mod, have been reported previously [7]. In these experiments toroidal rotation was seen to increment in

\begin{tabular}{|r|l|l|l|l|l|l|}
\cline { 2 - 7 } \multicolumn{1}{c|}{} & \multicolumn{4}{c|}{ Diffusivity $\left(\mathrm{m}^{2} / \mathrm{s}\right)$} & \multicolumn{1}{c|}{ Convection $(\mathrm{m} / \mathrm{s})$} \\
\hline Regime & $\chi_{\text {EFF }}$ & \multicolumn{1}{c|}{$\mathbf{D}_{\mathbf{I}}$} & \multicolumn{1}{c|}{$\mathbf{D}_{\boldsymbol{\phi}}$} & $\mathbf{D}_{\text {NC }}$ & $\mathbf{V}_{\mathbf{I}}$ & $\mathbf{V}_{\mathbf{C}}$ \\
\hline $\mathrm{L}$ & 1.0 & 0.5 & $0.20-0.25$ & 0.002 & 0 & 0 \\
\hline EDA & 0.5 & $0.1-0.3$ & $0.05-0.1$ & 0.0035 & 10 & 0 \\
\hline ELM-free & 0.5 & 0.05 & 0.4 & 0.003 & $10-100$ & 10 \\
\hline
\end{tabular}

Table 1. Transport coefficients for the diffusion of energy $\left(\chi_{E F F}\right)$, impurities $\left(D_{I}\right.$ and $\left.V_{I}\right)$ and momentum $\left(D_{\Phi}\right.$ and $\left.V_{C}\right)$ are obtained from time dependent analysis of plasma profiles in different confinement regimes. Note that the calculated neoclassical diffusivity $\left(D_{N C}\right)$ for momentum is always far below the observed values.

the co-current direction as plasma pressure increased, reaching Mach numbers up to 0.3 in high performance H-mode plasmas. In recent experiments, the transport of momentum has been measured by observing the transient relaxation of the rotation profile to changes in plasma boundary conditions [8,9]. Figure 3 shows the time history of rotation at several different plasma radii in such an experiment. For enhanced $\mathrm{D}_{\alpha} \mathrm{H}$ modes (EDA), the change is seen first at the outermost radius followed progressively by radii in the plasma core. The final state has a flat rotation profile. By contrast, for ELM-free $\mathrm{H}$ modes, the final profile is peaked indicating strong inward convection of momentum. Transport coefficients for these discharges are obtained by comparisons with a model for momentum transport. The best match is shown by solid lines in figure 3 and the transport coefficients summarized in table 1. Comparisons with neo-classical values show that the processes which transport momentum are highly anomalous in these experiments. The observations demonstrate that momentum diffuses or is convected from the edge region into the core. When the magnetic topology of the discharges is changed from lower single null (LSN) to upper single null (USN), the rotation at low pressure (or

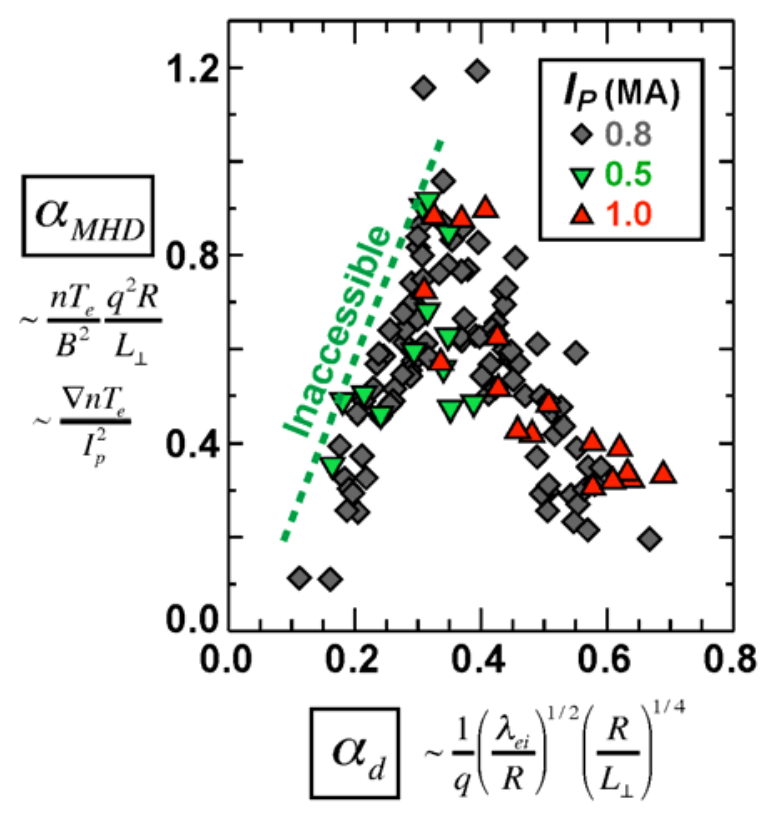

Fig 5. SOL profiles plotted as a normalized pressure gradient vs normalized collisionality map out a clear set of boundaries. 
input power) changed from nearly zero to strongly counter-current ( $\nabla B$ ion drift was down in all cases) [10]. Figure 4 shows the behavior of core rotation for low pressure plasmas as the distance between the primary and secondary separatrices (SSEP) is scanned from LSN (SSEP < 0 ) to USN (SSEP > 0). The extreme sensitivity to this parameter, a few mm difference causing the core rotation to change by $20 \mathrm{~km} / \mathrm{sec}$, is remarkable. The significance of these observations, the correlation with SOL flows and a hypothesis for the origin of boundary rotation is discussed in sections 4 and 5.

\section{Scrape-Off Layer Turbulence and Transport}

A set of fast scanning probes has allowed the determination of scrape-off layer (SOL) profiles, fluctuations and flows at various poloidal locations [11]. It is found that the profiles depend mainly on the local value of the normalized collisionality as predicted by theories for driftresistive-ballooning turbulence $[12,13,14]$. The boundary formed by experimental data (figure 5) conforms to boundaries found in simulations to define regions of significant change in edge transport. Observed fluctuation levels on the low-field (bad curvature) side of the plasma are found to be about an order of magnitude above those measured on the high-field (good
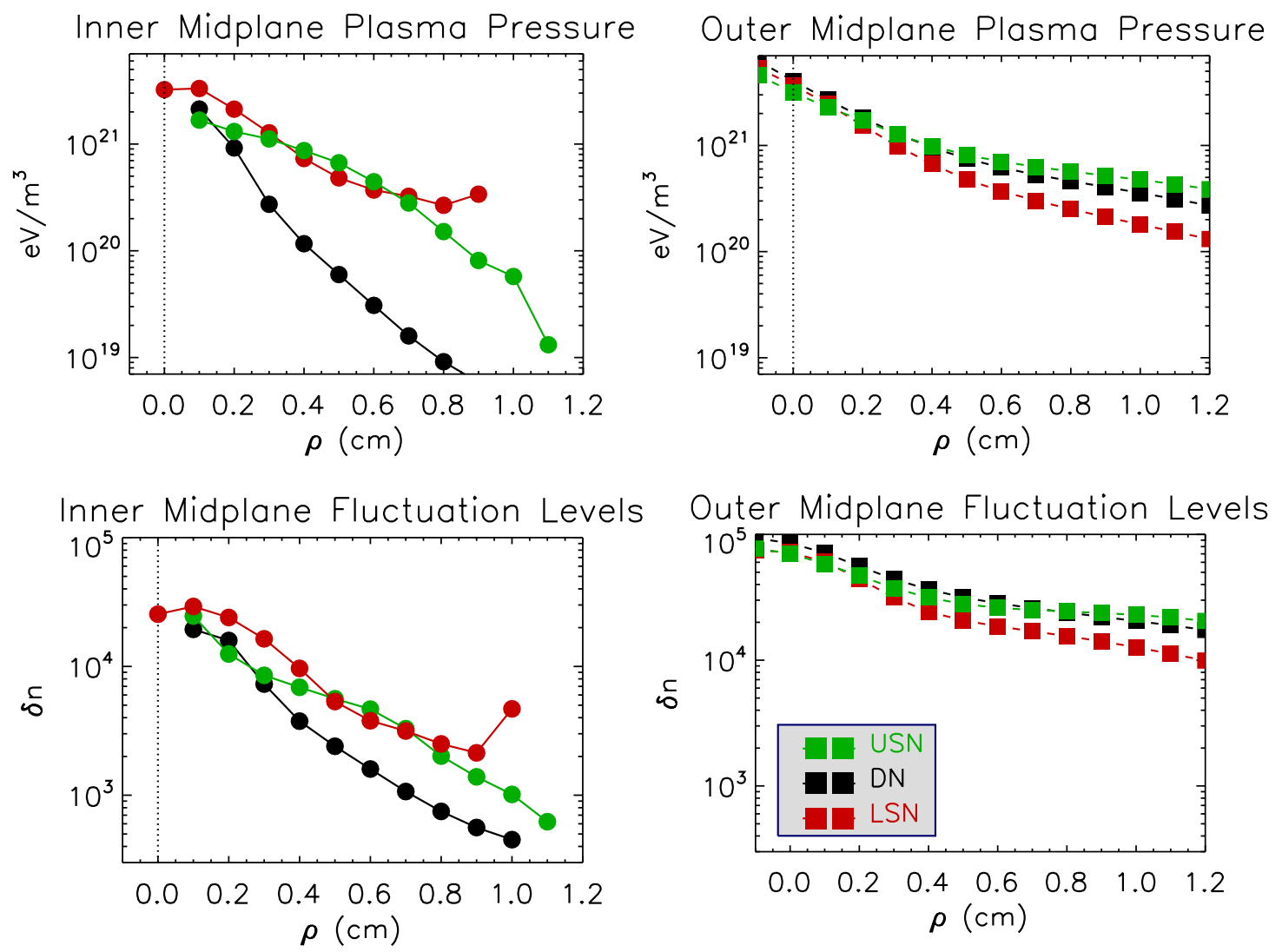

Fig 6. SOL pressure profiles and fluctuations measured at the inner (high-field) and outer (low-field) midplanes are compared. The dramatic drop in fluctuations on the inside and the drop in plasma pressure at that position for double-null discharges, confirms the ballooning nature of the cross-field transport and suggests that the innermidplane SOL is fuelled mainly by flows, also observed, from the outer-midplane. 


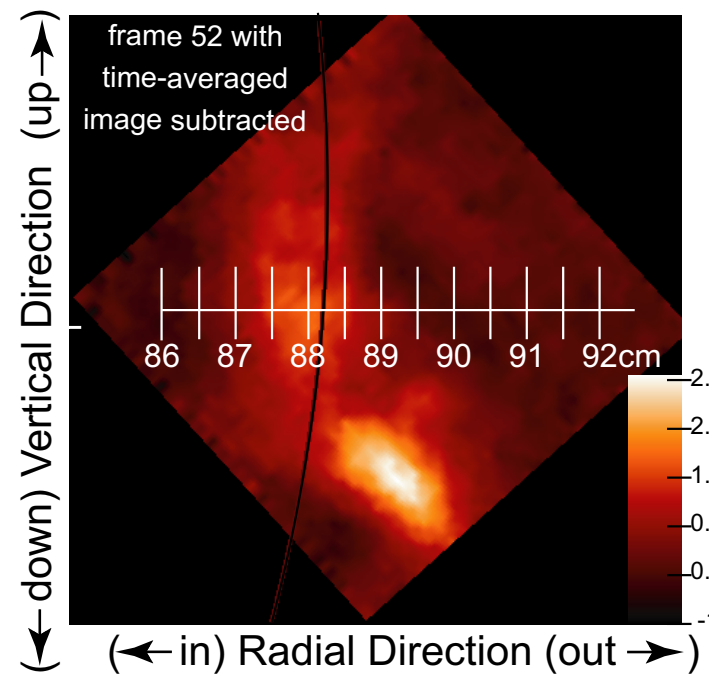

Fig 7a. Turbulence in the SOL, which has been visualized by a high-speed, high-resolution camera, is seen to be dominated by large $(\sim 1 \mathrm{~cm})$ structures.

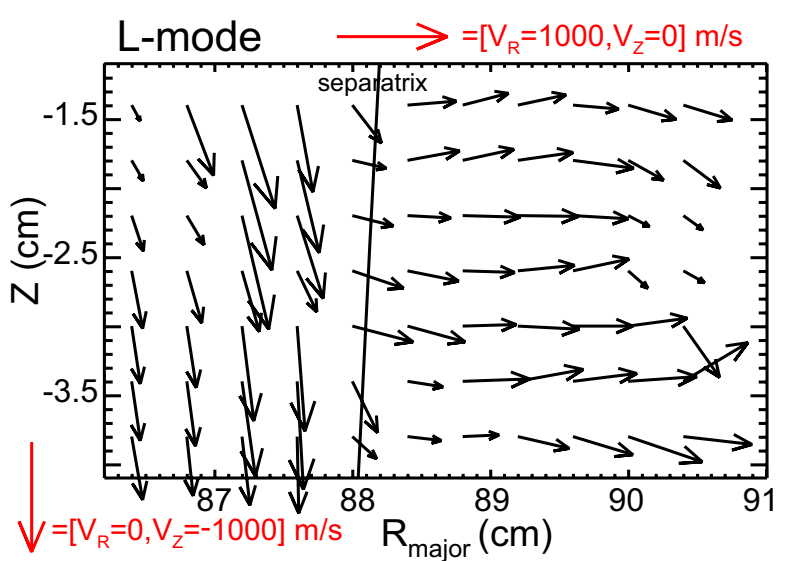

Fig 7b. Analysis of data from the high-speed camera reveal persistent phase velocities. Typically the fluctuations propagate in the poloidal direction inside the separatrix but pick up a large radial component in the SOL. The red arrows provide a scale for the plotted velocity vectors.

curvature) side demonstrating the ballooning (and finite $k_{\|}$) nature of the turbulence. Further, by varying the magnetic topology, it is found that the high-field side SOL is populated only via parallel transport from the connected bad curvature regions (figure 6) so that balanced double null discharges, which break this connection, have extremely low densities and pressures. The "re-symmetrization" transport in single null discharges is observed as near-sonic flows which reverse direction in the poloidal and toroidal sense as the discharge varies from LSN to USN.

Gas puff imaging (GPI), optical diagnostics which image this region with 1 - or 2-d detectors have been also used to investigate edge fluctuations [15]. These instruments view along magnetic field lines with toroidal localization provided by small, local gas puffs and have verified the poloidal asymmetries and the presence of large amplitude, field aligned structures (blobs, fig 7a) in the edge turbulence [16]. As seen in figure $7 b$, the phase velocities of the fluctuations are poloidal inside the separatrix. Outside the separatrix the fluctuations are identified with the blobs [17] and are observed to move radially with a variable poloidal component. In the SOL the radial velocities are typically $</ \sim 1 \mathrm{~km} / \mathrm{s}$. By careful

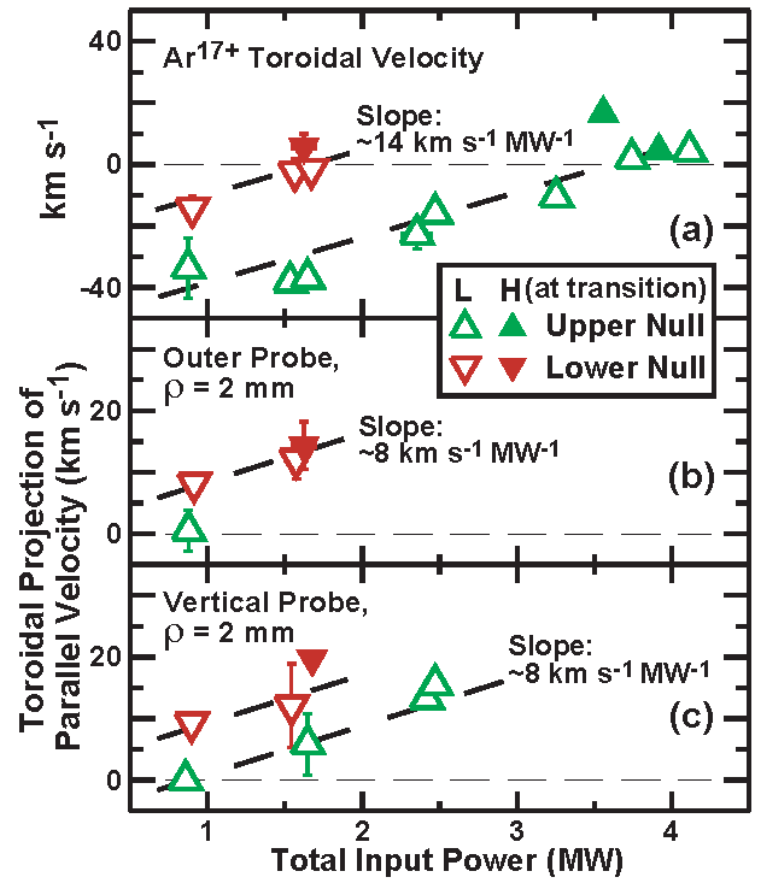

Fig 8. Both SOL and core flows increase in the co-current direction as input power (plasma pressure) increase. This adds to the topology dependent flows seen in fig. 4. 
adjustment of the plasma equilibrium, the scanning probe and GPI can be made to view the same flux tube, demonstrating the small, but finite $k_{\|}$[18]. The potential is found to have a dipole structure which straddles the density perturbation. As the density is raised toward the empirical limit, blobs can be observed well inside the separatrix and confirm observations of increased edge transport in this regime. Detailed studies with nonlinear simulations of the plasma edge have begun. Similarity comparisons with JET and DIII-D find dimensionlessly similar SOL profiles, suggesting that main chamber particle and power balance are likely the same on all three devices and suggesting a dominance of plasma physics over neutral dynamics [19].

\section{H-mode Threshold and Transport}

The dependence of the H-mode power threshold on plasma topology is a robust $0^{\text {th }}$ order effect which roughly doubles the power and temperature required for a transition when the $\nabla \mathrm{B}$ ion drift is away from a single magnetic x-point [20,21]. The correlation of self-generated plasma flows and topology discussed above has led to a novel explanation of the phenomenon which can be understood as the result of two rotation effects which add or subtract depending on topology [11]. The first effect is that SOL flows are driven by ballooning transport in a direction determined by topology and couple across the separatrix to the core plasma. The connection between SOL and core flows can be seen directly in C-Mod where complications from external momentum sources are not present. As shown in figure 3, momentum is observed to be transported inward from the outside to the inside of the plasma. Moreover, as seen in figure 4, both the SOL and core flows show the same quantitative dependence on edge topology. The second effect is determined by momentum transport and increases rotation monotonically in the co-current direction with plasma pressure. For the unfavorable topology, where the $\nabla \mathrm{B}$ ion drift is away from the $\mathrm{x}$-point, the discharge begins "farther" from the transition point and thus requires a higher plasma pressure than for the favorable topology (fig. 8). The H-mode threshold can be understood as dependent on a critical edge flow or flow shear which requires very different input powers for the two cases. The correlation between the L-H threshold, rotation, and the magnetic topology is clearly seen in fig. 9. The sensitivity to geometry may explain some of the large variation in threshold power reported for double-null discharges. It is interesting to note that limited plasmas, if the contact point is close to the lower divertor, have the same flow patterns and the same low power threshold as LSN diverted discharges [22].

Quantitative comparisons have been made to analytic models for the L-H transition [23]. This work, which is based on earlier fluid simulations [24] depends on the growth of zonal flows (and

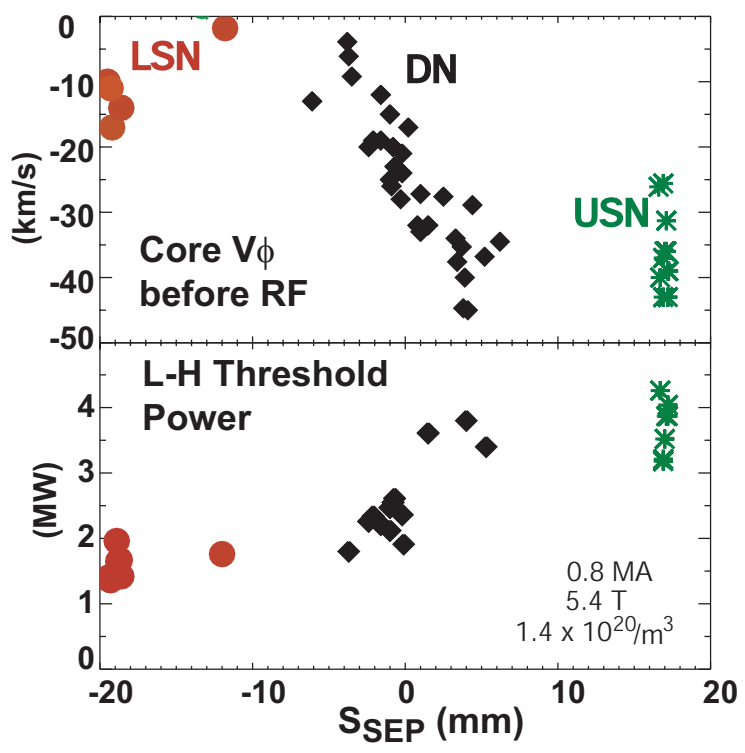

Fig 9. Both core rotation in the L-mode target plasma and the L-H threshold power are seen to depend strongly on ssep, a parameter which characterizes the magnetic topology. 
thus turbulence suppression) due to finite $\beta$ drift waves and leads to the threshold criterion: $\Theta \equiv \frac{T_{e}}{\sqrt{L_{n}}}>0.45 \frac{B_{T}^{2 / 3} Z_{E F F}^{1 / 3}}{\left(R A_{i}\right)^{1 / 6}} \quad$ where $\mathrm{L}_{\mathrm{n}}$ is the density scale length and $\mathrm{R}$ is the major radius in meters, $\mathrm{B}_{\mathrm{T}}$ is the toroidal field in Tesla and $A_{i}$ is the atomic mass of the plasma ions. Figure 10 tests the dependence of the theoretical threshold on toroidal field by showing a comparison of the predicted values to experimental data [25]. The $\mathrm{B}_{\mathrm{T}}$ scaling can be seen to be approximately correct, though the theory values are somewhat high at least for the normal topology and with $\nabla \mathrm{B}$ ion drift toward the $\mathrm{x}$-point. The single experimental datum for the unfavorable topology suggests that the prediction "splits the difference", perhaps appropriate for a theory which does not address the topology dependence.

Studies of the enhanced $\mathrm{D}_{\alpha} \mathrm{H}$-modes (EDA) have focused on accessibility conditions and the nature of the quasi-coherent (QC) mode, which is apparently the source of the increased edge particle transport which brings this regime to steady state without large ELMs [26]. EDA discharges are found to predominate at higher q, $\delta$ and collisionality [26,27]. As these parameters decrease, the QC mode becomes weaker, broader in frequency and less effective in transporting impurities leading to a gradual transition to the transient ELM-free regime. With strong heating at somewhat lower densities, a regime with higher plasma pressure and lower collisionality is obtained which is dominated by small, irregular, "Type II" ELMs. Comparisons of this ELM regime with the ELITE code demonstrate general agreement with the stability boundaries for intermediate $\mathrm{n}$ peeling-ballooning modes $[27,28]$. The QC mode has been tentatively identified as a resistive ballooning mode which is modified by the presence of the x-point geometry [2932]. Simulations reproduce the mode wavelength and operational boundaries. Additional observations of the QC mode have been made with beam emission spectroscopy (BES), GPI, electrostatic and magnetic probes and with an enhanced PCI system. The latter diagnostics demonstrated conclusively that there are no coherent high-frequency companions to the QC mode in contradiction to earlier BOUT predictions. Attempts to attain the EDA regime were made via dimensionless identity scaling with DIII-D and ASDEX-upgrade. In both cases, if the top of the pedestal was matched, the entire pedestal profile was found to scale as well [33]. Quasi-coherent fluctuations were observed in these regimes, but were apparently too weak to bring the discharges into steady state without ELMs. Dimensionless scaling experiments were also carried out to match discharges from JFT-2M which has reported EDA-like discharges in what they have called HRS, for high recycling steady regime [34]. Matching all parameters except the aspect ratio, these experiments resulted in EDA discharges in C-Mod. The EDA/ELM-free boundary in q- ${ }^{*}$ space matched the reported JFT-2M results as well [35]. 
Because of the strong correlation between edge and core parameters, the scaling of $\mathrm{H}$ mode pedestal widths has a direct impact on the performance predicted for future fusion devices like ITER. With this motivation, a set of high-resolution diagnostics has been employed on C-Mod to investigate the scaling of pedestal widths and gradients. Density and temperature pedestals are in the range $2-6 \mathrm{~mm}$ with the temperature profile slightly wider than the density. These widths are on the order of the poloidal gyroradius and scale with $1 / \mathrm{I}_{\mathrm{P}}$, but otherwise do not follow that scaling closely [36]. Unlike results in other devices, the density profile width in C-Mod increases with triangularity leading to somewhat lower pressure gradients at high triangularity. This may be the result of increased particle transport from the QC mode in C-Mod, which is stronger at high triangularity and is unlike the case of ELMy discharges where the width is expected to be set strictly by MHD

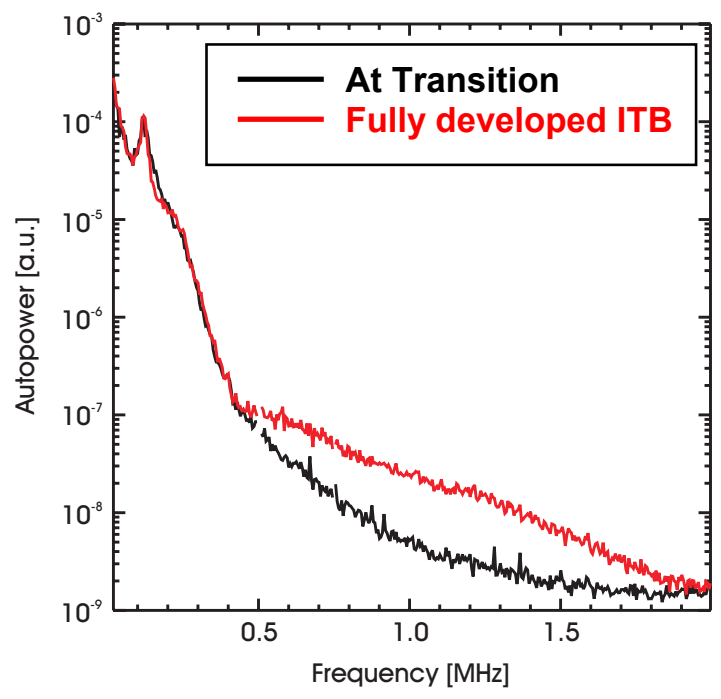

Fig 12. An increase in high-k fluctuations $\left(k_{\theta} \sim 8 \mathrm{~cm}^{-1}, k \rho_{s} \sim 1\right)$ is observed with PCI as the ITB discharge develops. These fluctuations may be the signature of the TEM turbulence predicted by gyrokinetic simulations.

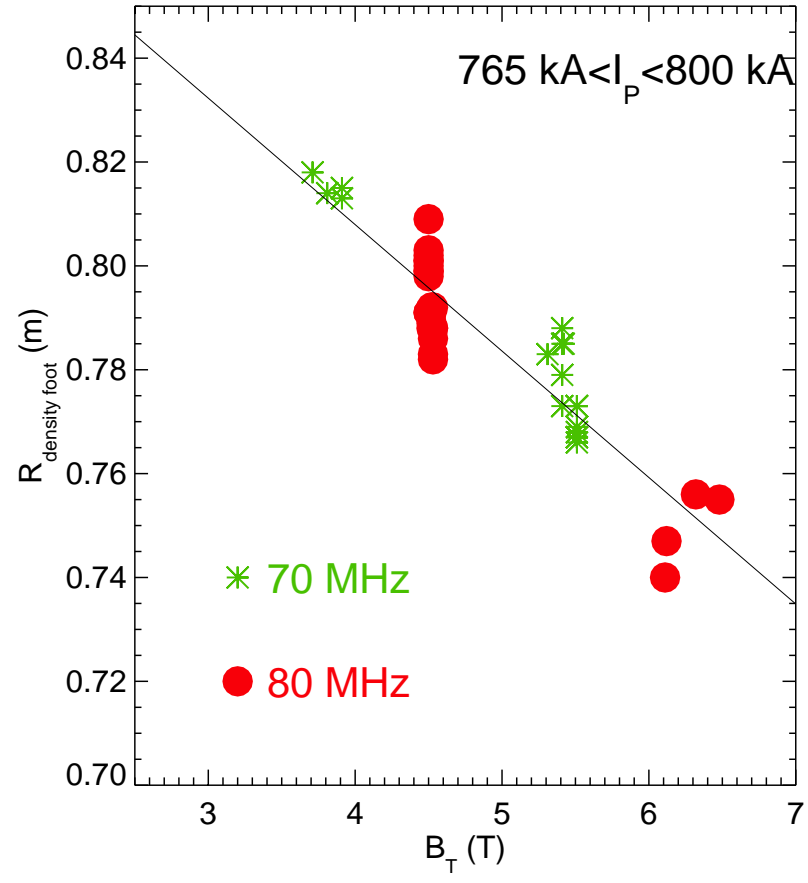

Fig 11. The location of the ITB foot is seen to scale with $B_{T}$. While strong correlations are observed with $B_{T}$ itself, it is not clear yet if the dependence is through the field, safety factor or magnetic shear.

stability limits. The density profile width shows little direct dependence on plasma density and based on studies with the kinetic neutral transport code $\mathrm{KN} 1 \mathrm{D}$, there is no indication of a trend with neutral penetration [37]. This result is supported by the dimensionless identity experiments discussed above. Profile similarity was obtained when the plasma physics parameter $\mathrm{na}^{2}$ was matched rather than the atomic physics parameter na (where a is the tokamak minor radius).

\section{Control of Internal Transport Barriers}

Research into internal transport barriers (ITB) has focused on control of the barrier strength, and location. The most robust method for producing plasmas with ITBs in C-Mod has been by off-axis ICRF heating. When the resonance location reaches about $\mathrm{r} / \mathrm{a} \sim 0.5$ on either the low or high-field side, a transport barrier develops and the plasma density 


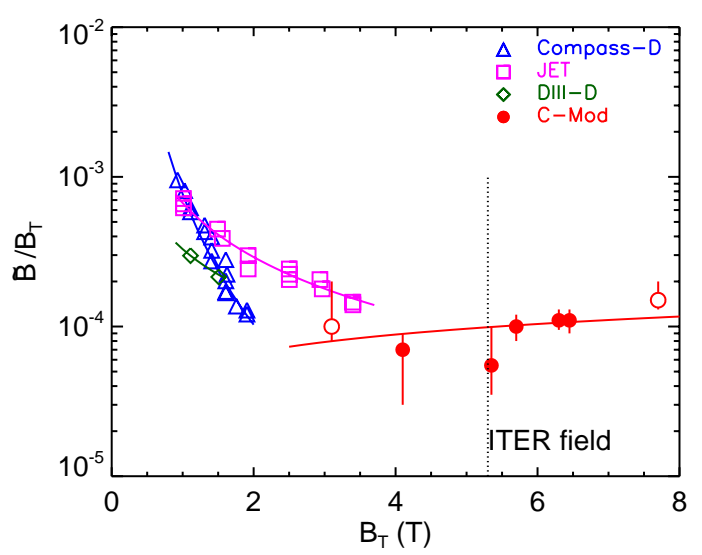

Fig 13. Scaling of the locked mode threshold from inter-machine comparisons allow extrapolation to ITER. Compass, DIII-D and JET data are from reference [49]. becomes strongly peaked [38,39]. Local analysis of transport for these discharges shows that energy diffusivity drops to or near the levels calculated for ion neo-classical transport [40]. Transient analysis of heat pulse propagation shows a narrow region of strongly increased incremental confinement, consistent with complete suppression of turbulence over that region [41]. With only off-axis heating, the plasma density continues to peak and impurities build up leading to a radiative collapse. However application of on-axis heating weakens the barrier and can arrest the density peaking and impurity accumulation [40]. The position of the barrier foot does not depend on the location of the ICRF resonance but can be moved to larger minor radius by lowering $\mathrm{B}_{\mathrm{T}}$ (fig 11). It is not entirely clear whether this an effect of $\mathrm{q}, \mathrm{q}^{\prime}$ or $\mathrm{B}_{\mathrm{T}}$ itself. It is found empirically that the barrier forms in a region with relatively weak shear at a radius where $\mathrm{q}$ is in the range 1.1 to $1.35[42,43]$.

Through comparison with simulations using the nonlinear gyro-kinetic code gs2, a picture of the formation and control mechanisms is emerging [44]. This work supports the hypothesis that the barrier forms when off-axis heating reduces $\mathrm{R} / \mathrm{L}_{\mathrm{T}}$ and begins to stabilize ITG turbulence. As the particle diffusivity drops, the Ware pinch begins to slowly peak the density profile. (While a small anomalous pinch cannot be ruled out, it is not present in the simulations and is not necessary to produce the observed peaking.) As the density gradient length shortens, $\mathrm{L}_{\mathrm{n}} / \mathrm{L}_{\mathrm{T}}$ drops and further stabilizes the ITG modes at which time little if any anomalous transport exists inside the barrier. At this point, driven by the density gradient, TEMs begin to go unstable and account for a modest increase in particle transport. In all of these dynamics, ExB stabilization is not dominant, at most playing a minor role during the onset of the ITB [45]. Control of the barrier is achieved with central heating via the $\mathrm{T}^{3 / 2}$ dependence of the TEM turbulent diffusivity which eventually balances

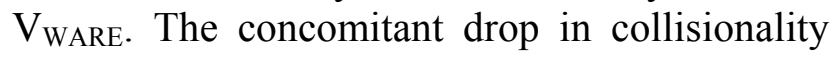
plays only a minor role. Quantitative agreement is found between the experimental measurements and predictions for particle transport which include a nonlinear upshift for the TEM threshold in $\mathrm{R} / \mathrm{L}_{\mathrm{N}}$ analogous to the

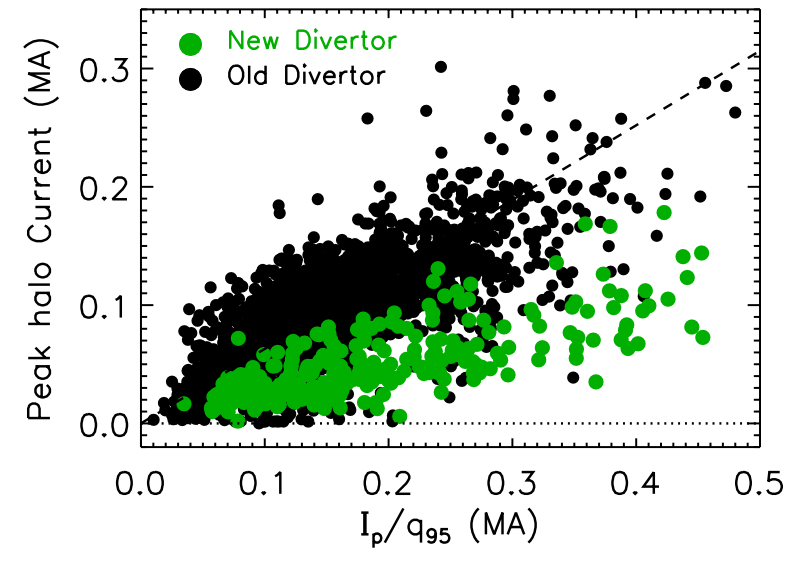

Fig 14. With the modified inner divertor, halo currents have dropped by about a factor of 2. The effect can be understood qualitatively from the change in plasma-wall contact geometry during a vertical displacement event. 
Dimits shift [46]. Fluctuations which may be due to the TEM turbulence at $k \rho_{\mathrm{s}} \sim 0.3-1$ are observed by PCI as the ITB develops (fig 12).

\section{Locked Modes, Disruptions and Fast Particle Modes}

A set of external non-axisymmetric control coils has been used to estimate the intrinsic error fields and locked mode threshold [47,48]. Intrinsic error fields as inferred from these experiments are consistent in amplitude and phase with known non-axisymmetries in the coils and buswork. The threshold is found to be roughly linear in density, with typical magnitudes for the $m=2, n=1$ component normalized to the total field of $10^{-4}$. As seen in fig 13, this threshold is similar to what is observed in larger low-field devices and suggests that locked modes should not be any more problematic for ITER than for existing devices. To definitively determine the field and size scaling, coordinated experiments on JET, DIII-D and C-Mod have been carried out. A dimensionless match is obtained within errors, which arise mainly from uncertainties in the amplitude and spectra of the intrinsic error fields. Locked modes lead to braking of toroidal rotation, modification of sawtooth activity, reduction of energy and particle confinement and often to disruptions. Reduction of error fields by use of the control coils suppressed locked modes and allowed operation of C-Mod at 2 MA.

A new inboard divertor with extensive diagnostics to measure halo and eddy currents has been installed on C-Mod with a geometry modified to allow production of discharges with higher triangularity. With the new divertor, the magnitude of halo currents was reduced by about a factor of 2 (fig 14) compared to the old configuration [50]. This reduction may be the direct result of the change in geometry; with the new configuration, the plasma does not contact the inner divertor until very late in a vertical displacement event (VDE), by which time most of the current has dissipated. Halo currents are also less toroidally asymmetric than with the previous divertor. The sensitivity of the halo current strength and symmetry to details of the divertor shape is of significance in the design of future machines and is currently the subject of investigation via non-linear extended-MHD simulations.

A pair of MHD antennae was installed in CMod to probe the stability of global MHD and fast particle modes [51]. With strong ICRF heating during the current rise, Alfven cascades are observed (fig 15) which indicate flat or slightly reversed shear during this period with $\mathrm{q}_{\min } \sim 3$. The density perturbation from these cascades is also observed with PCI.

ICRF driven TAE modes are seen during 'monster' sawteeth with moderate $\mathrm{n} \sim$ 4-10.

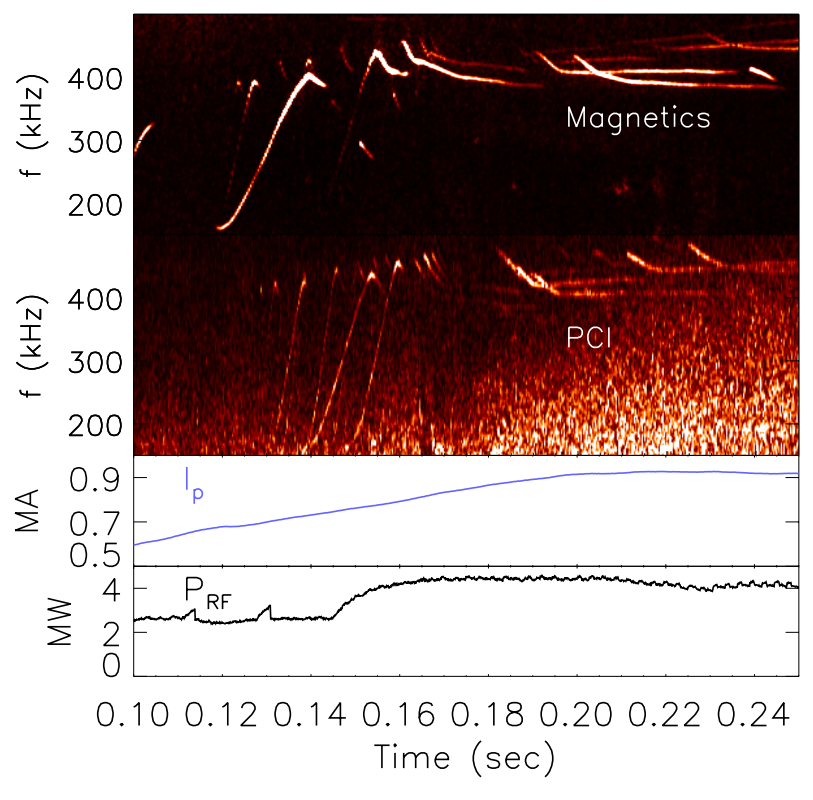

Fig 15. Alfven cascades are observed in magnetic and density fluctuations in plasmas with strong heating during the current ramp. A $q$ profile with flat or reversed shear and $q_{M I N} \sim$ 3 is inferred. 
Multiple modes are typically observed with frequencies chirping down from $750-650 \mathrm{kHz}$ and often coalescing just before the sawtooth collapse.

\section{Facility Upgrades and Future Plans}

Over the next two years, a number of facility upgrades on C-Mod will enhance its ability to address issues critical to fusion science and engineering. The major themes for that research will be support for the burning plasma program (ITER) and advanced tokamak studies in reactor relevant regimes, $\mathrm{T}_{\mathrm{i}} \sim \mathrm{T}_{\mathrm{e}} ; \mathrm{t}_{\text {pulse }}>\mathrm{t}_{\mathrm{L} / \mathrm{R}}$; no core particle or momentum source; and high power densities handled by a metal first wall. The most significant upgrade to C-Mod capabilities will be the addition of Lower Hybrid Current Drive (LHCD). This new system operates at $4.6 \mathrm{GHz}$ and features four 24-waveguide arrays with independent phasing of guides for good control of launched spectra, which will be important for current profile control in a range of advanced operating scenarios. The project will begin experiments in 2005 with $3 \mathrm{MW}$ source power and one launcher. A second launcher and a total of $4 \mathrm{MW}$ source power is planned for the future. ACCOME modeling based on actual plasma profiles predicts substantial current drive far off axis $(r / a \sim 0.75)$. This will enable exploration of the influence of $j(r)$ on transport barriers, and investigation of both non-inductive and "hybrid" scenarios. To further studies of high-Z first wall materials, a set of tungsten brush tiles is being developed for installation and testing on CMod. These tiles are comprised of $1 / 8$ " diameter $\mathrm{W}$ rods inserted into an inconel base. Tungsten has a $3200{ }^{\circ} \mathrm{C}$ melting temperature, $600{ }^{\circ} \mathrm{C}$ higher than molybdenum, providing additional operating margin before melting. Such tiles are prototypical of the proposed ITER divertor. In order to provide a means for active density control, a divertor cryopump is being developed, providing up to $25,000 \mathrm{l} / \mathrm{s}$ of pumping speed. A toroidal loop of liquid nitrogen and helium-cooled cryopanels will be placed on the inboard side of the upper divertor chamber, shielded by divertor tiles. The system will be optimized for pumping during unbalanced doublenull discharges (lower x-point dominant). This scenario maintains high neutral pressures in the upper divertor for pumping while sending SOL heat fluxes predominantly to the inclined target plates of the lower divertor. The concept also enables a dynamic regulation of the pumping throughput via upper x-point control. A long-pulse diagnostic neutral beam will soon be installed for use with the MSE, BES and CXRS diagnostics. The beam was built at the Budker Institute of Nuclear Physics in Novosibirsk, Russia, and will provide up to 8 amperes of ion current from the source at energies up to $55 \mathrm{keV}$. The beam pulse length can be up to $1.5 \mathrm{~s}$ steady state, or up to $3.0 \mathrm{~s}$ with $50 \%$ duty cycle modulation. A new long-life arc discharge ion source was developed in order to provide a high fraction $(>80 \%)$ of full-energy hydrogen ions, which is desirable for MSE.

\section{Acknowledgements}

This work was supported by the US Department of Energy.

\section{References}

[1] S.J. Wukitch, et al., Plasma Phys. and Controlled Fusion 46, 1479 (2004).

[2] E. Nelson-Melby, et al., Phys. Rev. Lett. 90, 155004 (2003). 
[3] Y. Lin, et al., Phys. Plasmas 11, 2466 (2004).

[4] F.W. Perkins, Nucl. Fusion 17, 1197 (1977).

[5] J.C. Wright, et al., Phys. Plasmas 11, 2473 (2004).

[6] E.F. Jaeger, et al., Phys. Rev. Lett. 90, 195001 (2003).

[7] J.E. Rice, et al., Nucl. Fusion 38, 75 (1998).

[8] W.D. Lee, et al., Phys. Rev. Lett. 91, 205003 (2003).

[9] J.E. Rice, et al., Nucl. Fusion 44, 379 (2004).

[10] J.E. Rice, et al., This conference EX/6-4.

[11] B. LaBombard, et al., Nucl. Fusion 44, 1047 (2004).

[12] B.N. Rogers and J.F. Drake, Phys. Rev. Lett. 79, 229 (1997).

[13] B.D. Scott, Plasma Phys. and Controlled Fusion 39, 1635 (1997).

[14] B. LaBombard, et al., Bull. Am. Phys. Soc., 48 no. 7, 52(2003).

[15] S. Zweben, et al., Phys. Plasmas 9, 1981 (2002).

[16] J. Terry, et al., Phys. Plasmas 10, 1739 (2003).

[17] J. Terry et al., This conference, EX/P4-12.

[18] O. Grulke, et al., submitted to Phys. Plasmas.

[19] B. Lipschultz, et al., submitted to Plasma Phys. and Controlled Fusion.

[20] ASDEX group, Nucl. Fusion 29, 1959 (1989).

[21] A. Hubbard, et al., Plasma Phys. and Controlled Fusion 40, 689 (1998).

[22] LaBombard et al., - submitted to Phys. Plasmas.

[23] P.N. Guzdar, et al., Phys. Rev. Lett. 89, 265004 (2002).

[24] B.N. Rogers, J.F. Drake, A. Zeiler, Phys. Rev. Lett. 81, 4396 (1998).

[25] A. Hubbard, et al., Plasma Phys. Cont. Fusion 46, A95 (2004).

[26] M. Greenwald, et al., Phys. Plasmas 6, 1943 (1999).

[27] D. Mossessian, et al, Phys. Plasmas, 10, (2003).

[28] H.R. Wilson, P.B. Snyder, G.T.A. Huysmans and R. L. Miller, Plasma Phys. Cont. Fusion 9, 1277 (2002).

[29] B.N. Rogers, J.R. Drake, A. Zeiler, Phys. Rev. Lett. 81, 4396 (1998).

[30] X. Xu, et al., Phys. Plasmas 7, 1951 (2000).

[31] A. Mazurenko, et al., Phys. Rev. Lett. 89, 225004 (2002).

[32] W.M. Nevins, et al., IAEA FEC, Lyon, paper TH/P3-07 (2002).

[33] D. Mossessian, et al., Plasma Phys. Cont. Fusion 42, A255 (2000).

[34] K. Kamiya, et al., Plasma Phys. Cont. Fusion 5A, A157 (2004).

[35] K. Kamiya, et al., Plasma Phys. Cont. Fusion 46, 1745 (2004).

[36] J.W. Hughes, et al., Phys. Plasmas 9, 3019 (2002).

[37] D. Mossessian, et al., submitted to Phys. Plasmas.

[38] C.L. Fiore, et al., Phys. Plasmas 8, 2023 (2001).

[39] J.E. Rice, et al., Nucl. Fusion 41, 277 (2001).

[40] J.E. Rice, et al., Nucl. Fusion 42, 510 (2002).

[41] S.J. Wukitch, et al., Phys Plasmas 9, 2149 (2002).

[42] C.L. Fiore, et al., Phys. Plasmas 11, 2480 (2004).

[43] J.E. Rice, et al., Nucl. Fusion 43, 781 (2003).

[44] D. Ernst, et al., Phys. Plasmas 11, 2637 (2004).

[45] M.H. Redi, et al., submitted to Phys. Plasmas.

[46] A.M. Dimits, et al., Phys. Plasmas 7, 969 (2000). 
[47] I.H. Hutchinson, et al.., This conference.

[48] S. Wolfe, et al., submitted to Phys. Plasmas.

[49] R.J. Buttery, et al., Nucl. Fusion 39, 1827 (1999).

[50] R. Granetz, et al, Nucl. Fusion 36, 545 (1996).

[51] J. Snipes, et al., submitted to Phys. Plasmas. 